\title{
Centrality in modular networks
}

\author{
Zakariya Ghalmane ${ }^{1,3}$, Mohammed El Hassouni ${ }^{1}$, Chantal Cherifi ${ }^{2}$ and Hocine Cherifi ${ }^{3^{*}}$ (i)
}

\author{
${ }^{*}$ Correspondence: \\ hocine.cherifi@u-bourgogne.fr \\ ${ }^{3}$ LE2I, UMR6306 CNRS, University of \\ Burgundy, Dijon, France \\ Full list of author information is \\ available at the end of the article
}

\begin{abstract}
Identifying influential nodes in a network is a fundamental issue due to its wide applications, such as accelerating information diffusion or halting virus spreading. Many measures based on the network topology have emerged over the years to identify influential nodes such as Betweenness, Closeness, and Eigenvalue centrality. However, although most real-world networks are made of groups of tightly connected nodes which are sparsely connected with the rest of the network in a so-called modular structure, few measures exploit this property. Recent works have shown that it has a significant effect on the dynamics of networks. In a modular network, a node has two types of influence: a local influence (on the nodes of its community) through its intra-community links and a global influence (on the nodes in other communities) through its inter-community links. Depending on the strength of the community structure, these two components are more or less influential. Based on this idea, we propose to extend all the standard centrality measures defined for networks with no community structure to modular networks. The so-called "Modular centrality" is a two-dimensional vector. Its first component quantifies the local influence of a node in its community while the second component quantifies its global influence on the other communities of the network. In order to illustrate the effectiveness of the Modular centrality extensions, comparison with their scalar counterparts is performed in an epidemic process setting. Simulation results using the Susceptible-Infected-Recovered (SIR) model on synthetic networks with controlled community structure allows getting a clear idea about the relation between the strength of the community structure and the major type of influence (global/local). Furthermore, experiments on real-world networks demonstrate the merit of this approach.
\end{abstract}

Keywords: Influential nodes; Centrality measures; Community Structure; SIR model

\section{Introduction}

Identifying the most influential nodes in a network has gained much attention among researchers in recent years due to its many applications. Indeed, these key nodes play a major role in controlling the epidemic outbreak [1], increasing the publicity on a new product [2], controlling the rumor spreading [3]. The most popular approach to uncover these central nodes is to quantify their influence using centrality measures. Various centrality measures have been proposed to quantify the influence of nodes based on their topological properties. Degree centrality, betweenness centrality, closeness centrality are among the most basic and the most widely used centrality measures.

The majority of real-world networks exhibit the modular organization of nodes, the socalled community structure [4-8]. Although there has been a tremendous effort regarding

(c) The Author(s) 2019. This article is distributed under the terms of the Creative Commons Attribution 4.0 International License (http://creativecommons.org/licenses/by/4.0/), which permits unrestricted use, distribution, and reproduction in any medium, provided you give appropriate credit to the original author(s) and the source, provide a link to the Creative Commons license, and indicate if changes were made. 
the definition of this property, there is no formal consensus on a definition that captures the gist of a community. It is intuitively apprehended as densely connected groups of nodes where individuals interact with each other more intensely than with those in the rest of the network. Therefore, communities are groups of nodes sharing some common properties and play similar roles in the interacting phenomenon within networks. Besides their various definitions, communities have been also found to show a number of interesting features such as the overlapping configuration of modules [9]. Some of the nodes can then be shared by multiple communities. Indeed, in some social networks, individuals can take part simultaneously in different groups, such as work colleagues, friends or family. In this work, we do not consider the overlaps between communities.

Previous works have shown that community structure has an important effect on the spreading process in networks [10-13]. However, classical centrality measures [14] do not take into account the influence of this major topological property on the spreading dynamics. In a modular network, we can distinguish two types of links $[15,16]$ that support the diffusion process: the links that connect nodes belonging to the same community (intra-community links or strong ties) and the links that bridge the communities (intercommunity links or weak ties). The former exercise a local influence on the diffusion process (i.e., at the community level), while the latter have a global influence (at the network level). Therefore, we believe that these two types of links should be treated differently. Indeed, the intra-community links contribute to the diffusion in localized densely connected areas of the networks, while the inter-community links allow the propagation to remote areas of the network. Suppose that an epidemic starts in a community, as it is highly connected, the intra-community links will tend to confine the epidemic inside the community, while the inter-community links will tend to propagate it to the other communities. As their role is quite different, we propose to represent the centrality of modular networks by a two-dimensional vector where the first component quantifies the intra-community (or local) influence and the second component quantifies the inter-community (or global) influence of each individual node in the network. To compute these components, we need to split the original network into a local and global network. The local network is obtained by removing all the inter-community links from the original network. The global network is obtained by removing all the intra-community links from the original network. Note that if the original network is made of a single connected component the global and local networks split into many connected components. Therefore, care must be taken to adapt the centrality definition to networks with multiple components. In the following, we restrict our attention to non-overlapping community structure (i.e. a node belongs to a single community). Furthermore, we consider undirected and unweighted networks for the sake of simplicity, but results can be easily extended to more general situations.

The proposed approach can be summarized as follows:

- Choose a standard centrality measure.

- Compute the local network by removing all the inter-community links from the modular network.

- Compute the Local component of the Modular centrality using the standard centrality.

- Compute the global network by removing all the intra-community links from the modular network.

- Compute the Global component of the Modular centrality using the standard centrality. 
As nodes need to be ranked according to their centrality values, it is necessary to adopt a strategy based on a combination of the two components of the Modular centrality. Various strategies, that include different levels of information about the community structure, may be used. As our main concern, in this paper, is to highlight the multidimensional nature of centrality in modular networks rather than devising optimal ranking methods, elementary strategies are evaluated. Two straightforward combination strategies (the modulus and the tangent of the argument of the Modular centrality) and a weighted linear combination of the components of the Modular centrality are investigated.

Experiments are conducted on modular synthetic networks in order to better understand the relative influence of the Local and Global component of the Modular centrality in the propagation process. Extensive comparisons with the standard centrality measures show that Modular centrality measures provide more accurate rankings. Simulations on real-world networks of diverse nature have also been performed. As their community structure is unknown, a community detection algorithm has been used. Results confirm that node rankings based on the Modular centrality are more accurate in terms of the epidemic size than those made by the standard centrality measures which have been designed for networks with no community structure.

The rest of the paper is organized as follows. Related modular-based measures are discussed in the next section. In Sect. 3, a general definition of the Modular centrality is given. In this framework, we present the extensions to modular networks of the most influential centrality measures (closeness, betweenness and eigenvector centrality). The experimental setting is described in Sect. 4. We report and analyze the results of the experiments performed on both synthetic and real-world networks in Sect. 5. Finally, the main conclusions are presented in Sect. 6 .

\section{Related works}

Ranking the nodes according to their centrality constitutes the standard deterministic approach to uncover the most influential nodes in a network. These measures rely usually on various network topological properties. However, the community structure of the network is rarely taken into consideration. Few researchers have paid attention to this property encountered in many real-world networks [10-13, 17-23]. In this section, we give a brief overview of the main deterministic methods that motivates our proposition.

a. Community centrality Newman proposed a slightly different formulation of the moduloarity. The Community centrality [24] is derived from the eigenvectors of the modularity matrix. Where the modularity matrix is divided into two projections. The first dimension represents the positive eigenvectors of the modularity matrix while the second dimension represents the negative ones. Thus, the modularity can be written in terms of these vectors as follows:

$$
Q=\sum_{k=1}^{c}\left|X_{k}\right|^{2}-\sum_{k=1}^{c}\left|Y_{k}\right|^{2},
$$

where $c$ is the number of communities. $X$ and $Y$ are the community eigenvectors in both dimensions. The $i$ th node in the community $k$ is represented by two vectors $x_{i}$ and $y_{i}$ (the $i$ th rows of $X_{k}$ and $Y_{k}$ respectively). 
The magnitude of a node vector $\left|x_{i}\right|$ specifies how central the node $i$ is in its community in terms of the number of connections. Thus, the node $i$ has a large positive contribution to the modularity when this measure is large. On the other hand, a higher value of $\left|y_{i}\right|$ means that the node $i$ has many connections to other nodes from foreign communities. Therefore, the Community centrality is defined to be equal to the vector magnitude $\left|x_{i}\right|$. It measures the strength with which a given node $i$ is assigned to its community. This measure was tested in the network of co-authorships between scientists. Results show that it is not very correlated with the degree centrality. Moreover, some nodes with high Community centrality measure have a relatively low degree. However, they have more connections with nodes of their communities. Thus, nodes with high Community centrality value play a central role in the spreading process in their local neighborhood.

b. Comm centrality N. Gupta et al. [25] proposed a degree-based centrality measure for networks with non-overlapping community structure. It is based on a non-linear combination of the number of intra-community links and inter-community links. The goal is to select nodes that are both hubs in their community and bridges between the communities. This measure gives more importance to community bridges. Indeed, the number of inter-community links is raised to the power of two. The comparison has been performed with deterministic and random immunization strategies using the SIR epidemic model and both synthetic and real-world networks. Nodes are immunized sequentially from each community in the decreasing order of their centrality value in their respective community. The number of nodes to be removed from a community are kept proportional to the community size. Results show that the Comm strategy is more effective or at least works as well as Degree and Betweenness centrality while using only information at the community level.

c. Number of neighboring communities centrality In a previous work [26], we proposed to rank the nodes according to the number of neighboring communities that they reach in one hop. The reason for selecting these nodes is that they are more likely to have a big influence on nodes belonging to various communities. Simulation results on different synthetic and real-world networks show that it outperforms Degree, Betweenness and Comm centrality in term of the epidemic size in networks with a community structure of medium strength (i.e. when the average number of intra-community links is of the same order than the number of inter-community links).

d. Community Hub-Bridge centrality We also proposed the Community Hub-Bridge centrality measure in [26]. It is based on the combination of the number of intracommunity links weighted by the size of the community and the inter-community links weighted by the number of neighboring communities. This measure tends to select preferentially nodes that can be considered as hubs inside large communities and bridges having many connections with various neighboring communities. According to experimental results, on both synthetic and real-world networks, this centrality measure is particularly suited to networks with strong community structure (i.e., when there are few inter-community links as compared to the number intra-community links). In this situation, it can identify effectively the most influential spreaders as compared to Degree, Betweenness, Comm and the Number of Neighboring Communities centrality measures. 
A variation of this centrality measure called the Weighted Community Hub-Bridge centrality has also been introduced. It is weighted such that, in networks with well-defined community structure, more importance is given to bridges (inter-community links), while in networks with weak community structure the hubs in the communities dominate. The goal is to target the bridges or the hubs according to the community structure strength. This measure has proved its efficiency as compared to the alternatives particularly in networks with weak community structure.

e. K-shell with community centrality Luo et al. proposed a variation of the K-shell decomposition for modular networks [27]. They suggest that the intra-community and the inter-community links should be considered separately in the K-core decomposition process. Their method works as follows:

(i) After the removal of nodes with intra-community links, the K-shell decomposition of the remaining nodes is computed. It is associated with an index of $k_{\text {core }}^{W}$.

(ii) After the removal of nodes with inter-community links, the K-shell of the remaining nodes is computed. It is associated with an index of $k_{\text {core }}^{S}$.

(iii) A new measure is then calculated and assigned to each node based on the linear combination of both $k_{\text {core }}^{W}$ and $k_{\text {core }}^{S}$ in order to find nodes that are at the same time bridges and hubs located in the core of the network.

Experiments have been performed using SIR simulations on Facebook friendship networks at US Universities. Results show that this strategy is more efficient in term of the epidemic size than the classical K-shell decomposition, the Degree and the Betweenness centrality measures.

f. Global centrality In [28], M. Kitromilidis et al. propose to redefine the standard centrality measures in order to characterize the influence of Western artist. Based on the idea that influential artists have connections beyond their artistic movement, they propose to define the centrality of modular networks by considering only the inter-community links. In other words, an influential artist must be related to multiple communities, rather than being strongly embedded in its own community. Considering a painter collaboration network where edges between nodes represent biographical connections between artists, they compared Betweenness and Closeness centrality measures with their classical version. Results show that the correlation values between the standard and modified centrality measures are quite high. However, the modified centrality measures allow to highlight influential nodes who might have been missed as they do not necessarily rank high in the standard measures.

All these works suggest that it is of prime interest to disentangle the local influence from the global influence in order to characterize a node centrality in modular networks. Indeed, these complementary types of influence may carry very different meanings and be more or less important in different situations. This is the reason why we propose to exploit the mesoscopic granularity level in order to extend the definition of the centrality measures that are agnostic about the community structure to modular networks. We propose to represent the centrality measures in modular networks as a two-dimensional vector made of its Local and Global component. If needed, these two components can be merged in a scalar value, but the combination can be made in multiple ways according to complementary available information about the network nature and topological properties. 


\section{Modular centrality}

Our main objective is to take into account the community structure in order to identify influential nodes. Indeed, in modular networks, a node has two types of influence: a local influence which is linked to its community features and a global influence related to its interactions with the other communities. Under this assumption, we provide a general definition of centrality in modular networks. We design a generic algorithm for computing the centrality of a node under this general definition. The Modular centrality extension can be naturally inferred from the various existing definitions of centrality designed for networks without community structure. To illustrate this process, we give the modular extensions of the most influential centrality measures (Betweenness, Closeness, and Eigenvector).

\subsection{Definitions}

\subsubsection{Local component of the Modular centrality}

Let's consider a network denoted as $G(V, E)$, where $V=\left\{v_{1}, v_{2}, \ldots, v_{n}\right\}$ and $E=\left\{\left(v_{i}, v_{j}\right) \backslash\right.$ $\left.v_{i}, v_{j} \in V\right\}$ denotes respectively the set of vertices and edges. Its non-overlapping community structure $\mathcal{C}$ is a partition into a set of communities $\mathcal{C}=\left\{C_{1}, \ldots, C_{k}, \ldots, C_{m}\right\}$ where $C_{k}$ is the $k$ th community and $m$ is the number of communities. The local network $G_{l}$ is formed by the union of all the disjoint modules of the network $G_{l}=\bigcup_{k=1}^{m} C_{k}$. These components are obtained by removing all the inter-community links between modules from the original network $G$. Each module represents a community $C_{k}$ denoted as $C_{k}\left(V_{k}, E_{k}\right)$. Where $V_{k}=\left\{v_{i}^{k} \backslash v_{i} \in V\right\}$ and $E_{k}=\left\{\left(v_{i}^{k_{1}}, v_{j}^{k_{2}}\right) \backslash v_{i}, v_{j} \in V\right.$ and $\left.k_{1}=k_{2}\right\}$, while $v_{i}^{k}$ refers to any node $v_{i}$ belonging to the community $C_{k}$.

For a selected centrality measure $\beta$, we define $\beta_{L}\left(v_{i}^{k}\right)$ as the Local centrality of the node $v_{i} \in V_{k}$. It is computed separately in each module $C_{k}$ of the local graph $G_{l}$.

\subsubsection{Global component of the Modular centrality}

Let's consider the network $G(V, E)$, the global network $G_{g}$ is formed by the union of all the connected components of the graph that are obtained after removing all the intra-community links from the original network $G(V, E)$. Let's suppose that $\mathcal{S}=$ $\left\{S_{1}, \ldots, S_{q}, \ldots, S_{p}\right\}$ is the set of the revealed connected components and $p=\left|\mathcal{C}^{\prime}\right|$ is the size of the set $\mathcal{S}$, the global network is defined by $G_{g}=\bigcup_{q=1}^{p} S_{q}$. Each component $S_{q}$ is denoted as $S_{q}\left(V_{q}, E_{q}\right)$. Where $V_{q}=\left\{v_{i}^{q} \backslash v_{i} \in V\right\}$ and $E_{q}=\left\{\left(v_{i}^{q_{1}}, v_{j}^{q_{2}}\right) \backslash v_{i}, v_{j} \in V\right.$ and $\left.q_{1}=q_{2}\right\}$, while $v_{i}^{q}$ refers to any node $v_{i}$ belonging to the component $S_{q}$. In this network, there may be some isolated nodes (i.e., nodes that are not linked directly to another community). These nodes are removed from $G_{g}$ in order to obtain a trimmed network formed only by nodes linked to different communities by one hop. Consequently, the set of nodes of $G_{g}$ is defined then by $V_{g}=\left\{v_{i} \in V \backslash\left|\mathcal{N}_{v_{i}}^{1}\right| \neq 0\right\}$. Where $\mathcal{N}_{v_{i}}^{n}$ is the neighborhood set of nodes reachable in $n$ hops. It is defined by $\mathcal{N}_{v_{i}}^{n}=\left\{v_{j} \in V \backslash v_{i} \neq v_{j}\right.$ and $\left.d_{G}\left(v_{i}, v_{j}\right) \leq n\right\}, d_{G}$ is the geodesic distance.

For a selected centrality measure $\beta$, we define $\beta_{G}\left(v_{i}^{q}\right)$ as the Global centrality of the node $v_{i} \in V_{q}$. It is computed over each connected component $S_{q}$ included in the global graph $G_{g}$. Remember that the Global centrality measure of the removed isolated nodes is set to 0 .

\subsubsection{Modular centrality}

It is a vector with two components. The first component quantifies the local influence of the nodes in their own community through the local graph $G_{l}$, while the second component measures the global influence of the nodes on the other communities through the 
connected components of the global graph $G_{g}$. The Modular centrality of a node $v_{i}$ is given by:

$$
B_{M}\left(v_{i}\right)=\left(\beta_{L}\left(v_{i}^{k}\right), \beta_{G}\left(v_{i}^{q}\right)\right) \quad k \in\{1, \ldots, m\} \text { and } q \in\{1, \ldots, p\},
$$

where $\beta_{L}$ and $\beta_{G}$ represent respectively the Local and Global centrality of the node $v_{i}$.

\subsection{Algorithm}

The Modular centrality is computed as follows:

Step 1. Choose a standard centrality measure $\beta$.

Step 2. Remove all the inter-community links from the original network $G$ to obtain the set of communities $\mathcal{C}$ forming the local network $G_{l}$.

Step 3. Compute the Local measure $\beta_{L}$ for each node in its own community.

Step 4. Remove all the intra-community links from the original network to reveal the set of connected components $\mathcal{S}$ formed by the inter-community links.

Step 5. Form the global network $G_{g}$ based on the union of all the connected component. Isolated nodes are removed from this network and their Global centrality value is set to 0 .

Step 6. Compute the Global measure $\beta_{G}$ of the nodes linking the communities based on each component of the global network.

Step 7. Add $\beta_{L}$ and $\beta_{G}$ to the Modular centrality vector $B_{M}$.

The pseudo-code of the algorithm to compute the Modular centrality is given in Algorithm 1.

\subsection{Modular extensions of standard centrality measures}

In order to illustrate the process allowing to extend a given centrality defined for a network without community structure to a modular network, we give as examples the modular definitions of the Betweenness, Closeness and Eigenvector centrality.

\subsubsection{Modular Betweeness centrality}

The modular Betweeness centrality takes into account separately paths that start and finish in the same community and those which starts and finish in different communities. For a given node $v_{i}$, it is represented by the following vector:

$$
B_{M}\left(v_{i}\right)=\left(\beta_{L}\left(v_{i}^{k}\right), \beta_{G}\left(v_{i}^{q}\right)\right) \quad k \in\{1, \ldots, m\} \text { and } q \in\{1, \ldots, p\},
$$

where:

$$
\begin{aligned}
& \beta_{L}\left(v_{i}^{k}\right)=\sum_{v_{s}, v_{t} \in C_{k}} \frac{\sigma_{s t}^{l}\left(v_{i}\right)}{\sigma_{s t}^{l}}, \\
& \beta_{G}\left(v_{i}^{q}\right)=\sum_{v_{s}, v_{t} \in S_{q}} \frac{\sigma_{s t}^{g}\left(v_{i}\right)}{\sigma_{s t}^{g}}
\end{aligned}
$$

$\beta_{L}$ measures the Betweenness centrality of nodes in their own community and $\beta_{G}$ measures the Betweenness centrality of nodes linking the communities. $\sigma_{s t}^{l}$ and $\sigma_{s t}^{g}$ count the number of shortest paths connecting nodes $v_{s}$ and $v_{t}$ based on the local and the global 


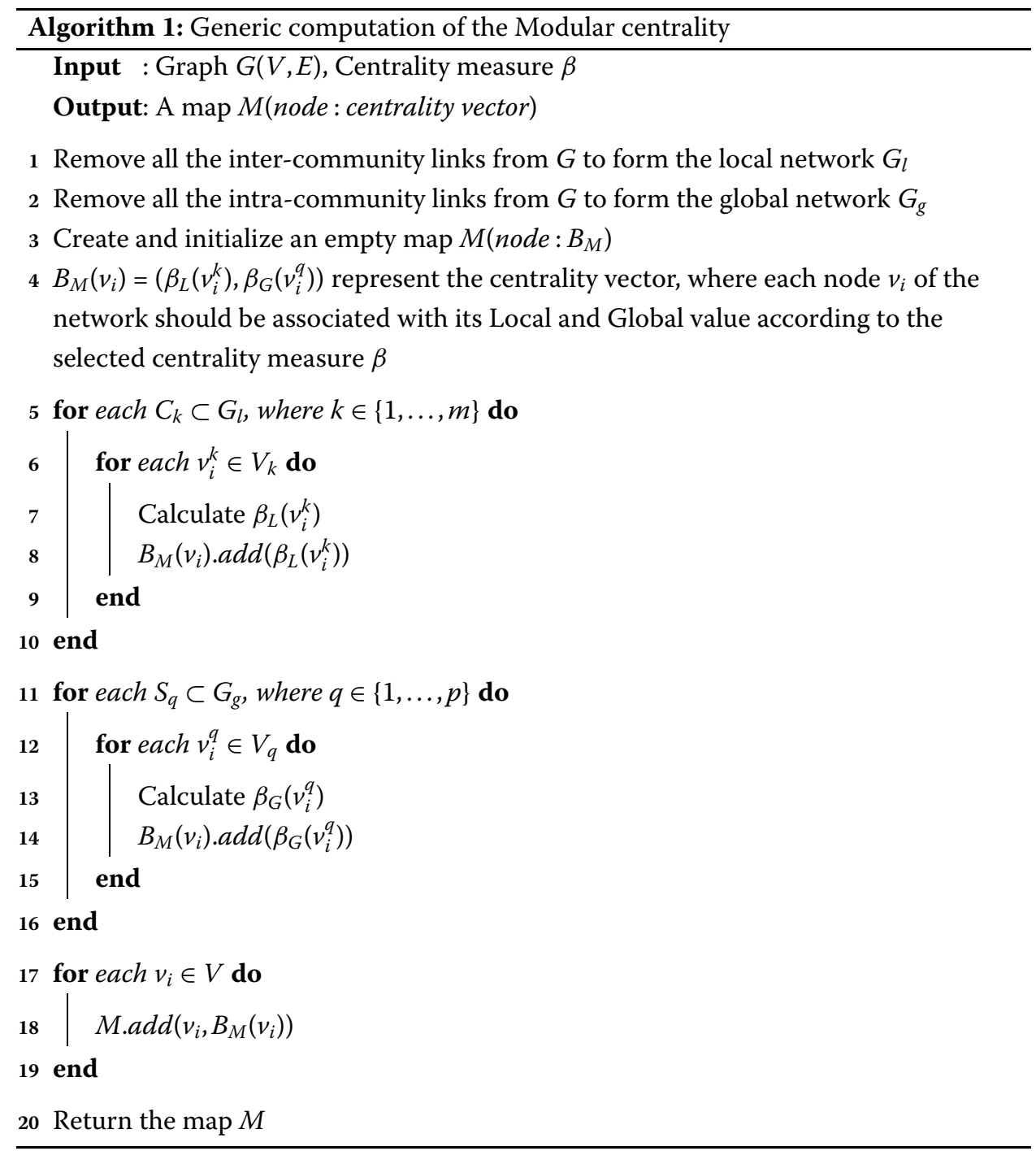

network respectively, while $\sigma_{s t}^{l}\left(v_{i}\right)$ and $\sigma_{s t}^{g}\left(v_{i}\right)$ represent the number of shortest paths connecting nodes $v_{s}$ and $v_{t}$ and passing through $v_{i}$ in the local and the global network respectively.

\subsubsection{Modular Closeness centrality}

Modular Closeness centrality considers separately the shortest distances of nodes originating from the same or from another community than the starting node $v_{i}$. It is defined as follows:

$$
B_{M}\left(v_{i}\right)=\left(\beta_{L}\left(v_{i}^{k}\right), \beta_{G}\left(v_{i}^{q}\right)\right) \quad k \in\{1, \ldots, m\} \text { and } q \in\{1, \ldots, p\},
$$

where:

$$
\beta_{L}\left(v_{i}^{k}\right)=\frac{1}{\sum_{v_{j} \in C_{k}} d_{i j}^{l}},
$$




$$
\beta_{G}\left(v_{i}^{q}\right)=\frac{1}{\sum_{v_{j} \in S_{q}} d_{i j}^{g}}
$$

$\beta_{L}$ and $\beta_{G}$ measure respectively the Local and Global component of the Modular Closeness centrality. $d_{i j}^{l}$ and $d_{i j}^{g}$ indicate the length of the geodesic from node $v_{i}$ to node $v_{j}$ based on the local and the global network respectively.

\subsubsection{Modular Eigenvector centrality}

Modular Eigenvector takes into account separately both the number and the importance of the neighbors belonging to the same community and those belonging to different communities to measure its centrality. The community based Eigenvector of a node $v_{i}$ is defined by the following vector:

$$
B_{M}\left(v_{i}\right)=\left(\beta_{L}\left(v_{i}^{k}\right), \beta_{G}\left(v_{i}^{q}\right)\right) \quad k \in\{1, \ldots, m\} \text { and } q \in\{1, \ldots, p\},
$$

where:

$$
\begin{aligned}
& \beta_{L}\left(v_{i}^{k}\right)=\frac{1}{\lambda} \sum_{v_{j} \in C_{k}} a_{i j} \beta_{L}\left(v_{j}^{k}\right), \\
& \beta_{G}\left(v_{i}^{q}\right)=\frac{1}{\lambda} \sum_{v_{j} \in S_{q}} a_{i j} \beta_{L}\left(v_{j}^{q}\right)
\end{aligned}
$$

$\beta_{L}$ and $\beta_{G}$ measure respectively the Local and Global component of the Modular Eigenvector centrality. $A=\left(a_{i, j}\right)$ is the network adjacency matrix, i.e. $a_{i, j}=1$ if vertex $v_{i}$ is linked to vertex $v_{j}, 0$ otherwise, and $\lambda$ is a constant.

\subsection{Toy example}

The toy example reported in Fig. 1 allows to illustrate the two types of influence that can occur in a modular network. For the sake of simplicity, we consider the Degree centrality measure (refer to Table 1). In this case, $v_{4}$ and $v_{11}$ are the most influential nodes as they have the highest degree value $\left(\beta\left(v_{11}\right)=\beta\left(v_{4}\right)=7\right)$. Even though they share the same degree value the influence they have on the other nodes of the network is not comparable. Indeed, their position in the network is quite different: $v_{11}$ is embedded in its community, while $v_{4}$ is at the border of its community. Inspecting the local and global networks give us a clear picture of their differences. As shown in the local network $G_{l}$, node $v_{11}$ is the most influential at the community level since it is linked to all the of nodes of its community $\left(\beta_{L}\left(v_{11}^{2}\right)=7\right)$, while $v_{4}$ is only linked to 3 nodes of its community $\left(\beta_{L}\left(v_{4}^{1}\right)=3\right)$. Actually,

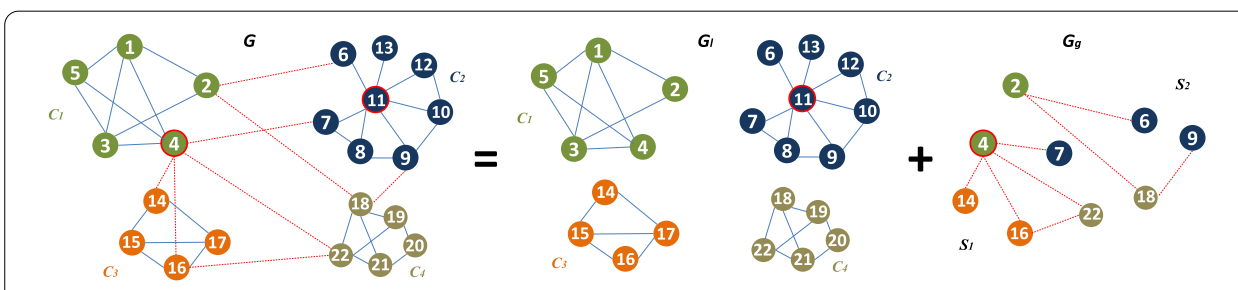

Figure 1 A toy example representing the Local network $\left(G_{l}\right)$ and the Global network $\left(G_{g}\right)$ associated to a modular network $(G)$ made of four non-overlapping communities 
Table 1 Standard Degree centrality, Global and Local Component of the Modular Degree Centrality of the nodes in the toy example

\begin{tabular}{llrrrrrrrrrr}
\hline Node ID & 4 & 11 & 18 & 22 & 1 & 2 & 3 & 16 & 5 & 7 & 8 \\
\hline$\beta$ & $\mathbf{7}$ & $\mathbf{7}$ & 5 & 5 & 4 & 4 & 4 & 4 & 3 & 3 & 3 \\
$\beta_{L}$ & 3 & $\mathbf{7}$ & 3 & 3 & 4 & 2 & 4 & 2 & 3 & 2 & 3 \\
$\beta_{G}$ & $\mathbf{4}$ & 0 & 2 & 2 & 0 & 2 & 0 & 2 & 0 & 1 & 0 \\
\hline \hline Node ID & 9 & 10 & 14 & 15 & 17 & 19 & 21 & 6 & 12 & 20 & 13 \\
\hline$\beta$ & 3 & 3 & 3 & 3 & 3 & 3 & 3 & 2 & 2 & 2 & 1 \\
$\beta_{L}$ & 2 & 3 & 2 & 3 & 3 & 3 & 3 & 1 & 2 & 2 & 1 \\
$\beta_{G}$ & 1 & 0 & 1 & 0 & 0 & 0 & 0 & 1 & 0 & 0 & 0 \\
\hline
\end{tabular}

both nodes $v_{1}$ and $v_{3}$ are more influential than node $v_{4}$ in the community $C_{1}$ with their higher Local Degree values. Looking at the Global network $G_{g}$, it appears clearly than node $v_{4}$ is the most influential node at the network level since it is connected to 4 nodes inside its component $\left(\beta_{G}\left(v_{4}^{1}\right)=4\right)$. These nodes belong to all the other communities of the network $\left(C_{1}, C_{2}\right.$ and $\left.C_{3}\right)$. Therefore, $v_{4}$ is more influential than node $v_{11}$ in the global network $G_{g}$ because of its ability to reach the different modules of the network as compared to $v_{11}$ which is influential only locally (in the community $C_{2}$ ).

To sum up, it can be noticed from this example that when we consider the Degree centrality, the community hubs are the most influential spreaders locally due to their ability to reach a high number of nodes in their own communities. The bridges which are linked to various communities are the most influential spreaders globally as they allow to reach a high number of communities all over the network.

\subsection{Modular centrality ranking strategies}

In order to rank the nodes according to their centrality, it is necessary to derive a scalar value from the Modular centrality vector. To do so, we can proceed in many different ways. In order to highlight the essential features of centrality in modular networks, we choose to consider three strategies. The first two are straightforward. Indeed, a simple way to combine the components of the Modular centrality is to use the modulus and the argument of this vector. The third strategy uses more information about the community structure in order to see if this can be beneficial.

The modulus $r$ of the modular vector $B_{M}$ of a node $v_{i}$ is defined by:

$$
r\left(v_{i}\right)=\left\|B_{M}\left(v_{i}\right)\right\|=\sqrt{\left(\beta_{L}\left(v_{i}^{k}\right)\right)^{2}+\left(\beta_{G}\left(v_{i}^{q}\right)\right)^{2}} \quad k \in\{1, \ldots, m\} \text { and } q \in\{1, \ldots, p\}
$$

The argument $\varphi$ of the modular vector $B_{M}$ of a node $v_{i}$ is defined as follows:

$$
\varphi\left(v_{i}\right)=\arctan \left(\frac{\beta_{G}\left(v_{i}^{q}\right)}{\beta_{L}\left(v_{i}^{k}\right)}\right) \quad k \in\{1, \ldots, m\} \text { and } q \in\{1, \ldots, p\}
$$

We propose to use the tangent of the argument because it has a higher range than the argument. It is defined by:

$$
\tan \left(\varphi\left(v_{i}\right)\right)=\frac{\beta_{G}\left(v_{i}^{q}\right)}{\beta_{L}\left(v_{i}^{k}\right)} \quad k \in\{1, \ldots, m\} \text { and } q \in\{1, \ldots, p\}
$$


Note that in these ranking strategies the information used about the community structure is very limited. As we expect that integrating more knowledge about the community structure in the combination strategy of the Modular centrality components may improve the efficiency of the ranking method, we also investigate the so-called "Weighted Modular measure". It is based on a linear combination of the components of the Modular centrality vector weighted by a measure of the strength of the communities.

The Weighted Modular measure $\alpha_{W}$ of a node $v_{i}$ is given by:

$$
\alpha_{W}\left(v_{i}\right)=\left(1-\mu_{C_{k}}\right) * \beta_{L}\left(v_{i}^{k}\right)+\mu_{C_{k}} * \beta_{G}\left(v_{i}^{q}\right),
$$

where $k \in\{1, \ldots, m\}, q \in\{1, \ldots, p\}$ and:

$$
\mu_{C_{k}}=\frac{\sum_{v_{i} \in C_{k}} k^{\text {inter }}\left(v_{i}^{k}\right)}{\sum_{v_{i} \in C_{k}} k\left(v_{i}^{k}\right)},
$$

where $\mu_{C_{k}}$ is the fraction of inter-community links of the community $C_{k}$.

$k^{\text {inter }}\left(v_{i}^{k}\right)$ is the number of inter-community links of node $v_{i}^{k}$ and $k\left(v_{i}\right)$ is the degree of node $v_{i}^{k}$.

The Weighted Modular measure works as follows:

- A community $C_{k}$, where the intra-community links predominate is densely connected and therefore it has a very well-defined community structure. If an epidemic starts in such a cohesive community, it has more chance to stay confined than to propagate through the few links that allows to reach the other communities of the network. In this case, priority must be given to local immunization. Consequently, more weight is given to the Local component of the Modular centrality $\beta_{L}$ to target the most influential nodes in the community since it is well separated from the other communities of the network.

- A community $C_{k}$ where the inter-community links predominate has a non-cohesive community structure. It is more likely that an epidemic starting in this community diffuses to the other communities through the many links that it shares with the other communities. Consequently, more weight is given to the Global component of the Modular centrality measure $\beta_{G}$ in order to target nodes that can propagate the epidemic more easily all over the network due to the loose community structure of $C_{k}$.

\section{Experimental setting}

In this section, we give some information about the synthetic and real-world dataset used in the empirical evaluation of the centrality measures. The SIR simulation process is recalled, together with the measure of performance used in the experiments.

\subsection{Dataset}

\subsubsection{Synthetic networks}

In order to generate artificial modular networks with controlled topological properties, the LFR benchmark is used [29]. It allows generating small-world networks with a powerlaw distributed degree and community size. The input parameters of the model are the number of nodes, the desired average and maximum degree, the exponents for the degree and the community size distributions, and the mixing coefficient. The mixing coefficient parameter $\mu$ value ranges from 0 to 1 . It represents the average proportion of links be- 
Table 2 LFR network parameters

\begin{tabular}{ll}
\hline Number of nodes & 4000 \\
Average degree & 7 \\
Maximum degree & 80 \\
Exponent for the degree distribution & 2.8 \\
Exponent for the community size distribution & 2 \\
Mixing parameter & $0.1,0.4,0.7$ \\
Community size range & {$[15200]$} \\
\hline
\end{tabular}

tween a node and the ones located outside of its community. This parameter allows for controlling the strength of the community structure. If its value is low, there are few links between the communities and they are well separated from each other. In the following, we designate this situation as "well-defined community structure". A high value of $\mu$ indicates a very loose community structure. Indeed, in this case, a node shares more links with nodes outside its community than with nodes inside its community. With $\mu$ values ranging from 0.2 to 0.45 , the community structure is referred as "community structure with medium cohesiveness". Experimental studies have shown that typical value of the degree distribution exponent in real-world networks varies in the range $2 \leq \gamma \leq 3$. Networks can have different size going from tens to millions of nodes. In addition, it is also difficult to characterize the average and the maximal degree since they are very variable. Consequently, we choose for these parameters some consensual values while considering also the computational aspect of the simulations. They are reported in Table 2.

\subsubsection{Real-world networks}

Although the LFR model produces pretty realistic networks, uncontrolled properties such as transitivity and degree correlation can deviate significantly from those observed in realworld networks [30]. Therefore, it is necessary to use real-world networks in the evaluation process. In order to cover a wide range of situations, we selected networks from various origin: online social networks, collaboration networks, technological networks, communication networks. All networks are undirected and unweighted. Experiments are performed on their largest connected component. The Louvain Algorithm is used to unveil the community structure of these networks. We choose this greedy optimization method for its simplicity. Furthermore, this popular algorithm has proved to be a good compromise between efficiency and complexity when used in many different types of networks $[31,32]$.

- Social networks: Four Samples of the Facebook Network are used. The ego-Facebook network collected from survey participants using the Facebook app. [33] and the Facebook friendship network at 3 US universities (Caltech, Princeton, Georgetown) collected by Traud et al. [34]. Nodes represent individuals (survey participant or members of the University), and edges represent online friendship links between two individuals. In the University network, in order to obtain data that are relevant for the spread of epidemic infections, only the relationship of individuals who live in the same dormitory or study the same major are considered.

- Communication network: The Email-Eu-core ${ }^{\mathrm{a}}$ network has been generated using email data from a large European research institution. The dataset contains only communication between institution members. Each node corresponds to an email address and an edge is established between two nodes $u$ and $v$, if at least one email has been exchanged between address $u$ and address $v$. 
Table 3 Description of the structural properties of the real-world networks. $N$ is the total numbers of nodes, $E$ is the number of edges. $\langle k\rangle, k_{\max }$ are respectively the average and the max degree. $C$ is the average clustering coefficient. $\alpha_{\text {th }}$ is the epidemic threshold of the network

\begin{tabular}{|c|c|c|c|c|c|c|}
\hline Network & $N$ & E & $\langle k\rangle$ & $k_{\max }$ & C & $\alpha_{\text {th }}$ \\
\hline ego-Facebook & 4039 & 88,234 & 43.69 & 1045 & 0.605 & 0.009 \\
\hline Caltech & 620 & 7255 & 43.31 & 248 & 0.443 & 0.012 \\
\hline Princeton & 5112 & 28,684 & 88.93 & 628 & 0.298 & 0.006 \\
\hline Georgetown & 7423 & 162,982 & 90.42 & 1235 & 0.268 & 0.006 \\
\hline Email-Eu-core & 986 & 25,552 & 33.24 & 347 & 0.399 & 0.013 \\
\hline Power-grid & 4941 & 6594 & 2.66 & 19 & 0.107 & 0.092 \\
\hline CR-QC & 4158 & 13,428 & 5.53 & 81 & 0.529 & 0.059 \\
\hline
\end{tabular}

- Technological network: Power-Grid ${ }^{\mathrm{b}}$ is a network containing information about the topology of the Western States Power Grid of the United States. An edge represents a power supply line. A node is either a generator, a transformer or a substation.

- Collaboration network: GR-QC (General Relativity and Quantum Cosmology) collaboration network has been collected from the e-print arXiv and covers scientific collaborations between authors of papers submitted to the General Relativity and Quantum Cosmology category. If an author $i$ co-authored a paper with author $j$, the graph contains an edge from $i$ to $j$. If the paper is co-authored by $k$ authors this generates a completely connected (sub)graph on $k$ nodes.

The basic topological properties of these networks are given in Table 3.

\subsection{SIR simulations}

To evaluate the efficiency of the centrality measures, we consider an epidemic spreading scenario using the Susceptible-Infected-Recovered (SIR) model [35]. In this setting, nodes can be classified into three classes: $S$ (Susceptible), I (Infected) and R (Recovered). Initially, all nodes are set as susceptible nodes. Then, a given fraction $f_{0}$ of the top-ranked nodes according to the centrality measure under test are set to the state infected. After this initial setup, at each iteration, each infected node affects one of its susceptible neighbors with probability $\alpha$. Besides, the infected nodes turn into recovered nodes with the recover probability $\sigma$. To better characterize the spreading capability, the value of the transmission rate $\alpha$ is chosen to be greater than the network epidemic threshold $\alpha_{\text {th }}$ given by [36]:

$$
\alpha_{\mathrm{th}}=\frac{\langle k\rangle}{\left\langle k^{2}\right\rangle-\langle k\rangle},
$$

where $\langle k\rangle$ and $\left\langle k^{2}\right\rangle$ are respectively the first and second moments of the degree distribution. The epidemic threshold values $\alpha_{\text {th }}$ for the networks used in this study are reported in Table 3. In all the experiments, we use the same value of the transmission rate $(\alpha=0.1)$. Naturally, it is much larger than the values of the epidemic threshold $\alpha_{\text {th }}$ of all the dataset. The value of the recovery probability is also constant $(\sigma=0.1)$. We choose this small value so that each infected node may have many chances to infect its neighbors with the probability of $\alpha$ before changing to the recovered status. The process continues until there is no more infected node in the network. Finally, when the spreading process stops, the number of nodes in the state "Recovered" $R$ is used to measure the spreading efficiency of the fraction of the initially infected nodes. The larger the value of $R$, the more influential the initially selected nodes. To ensure the effectiveness of the evaluation process, the results 


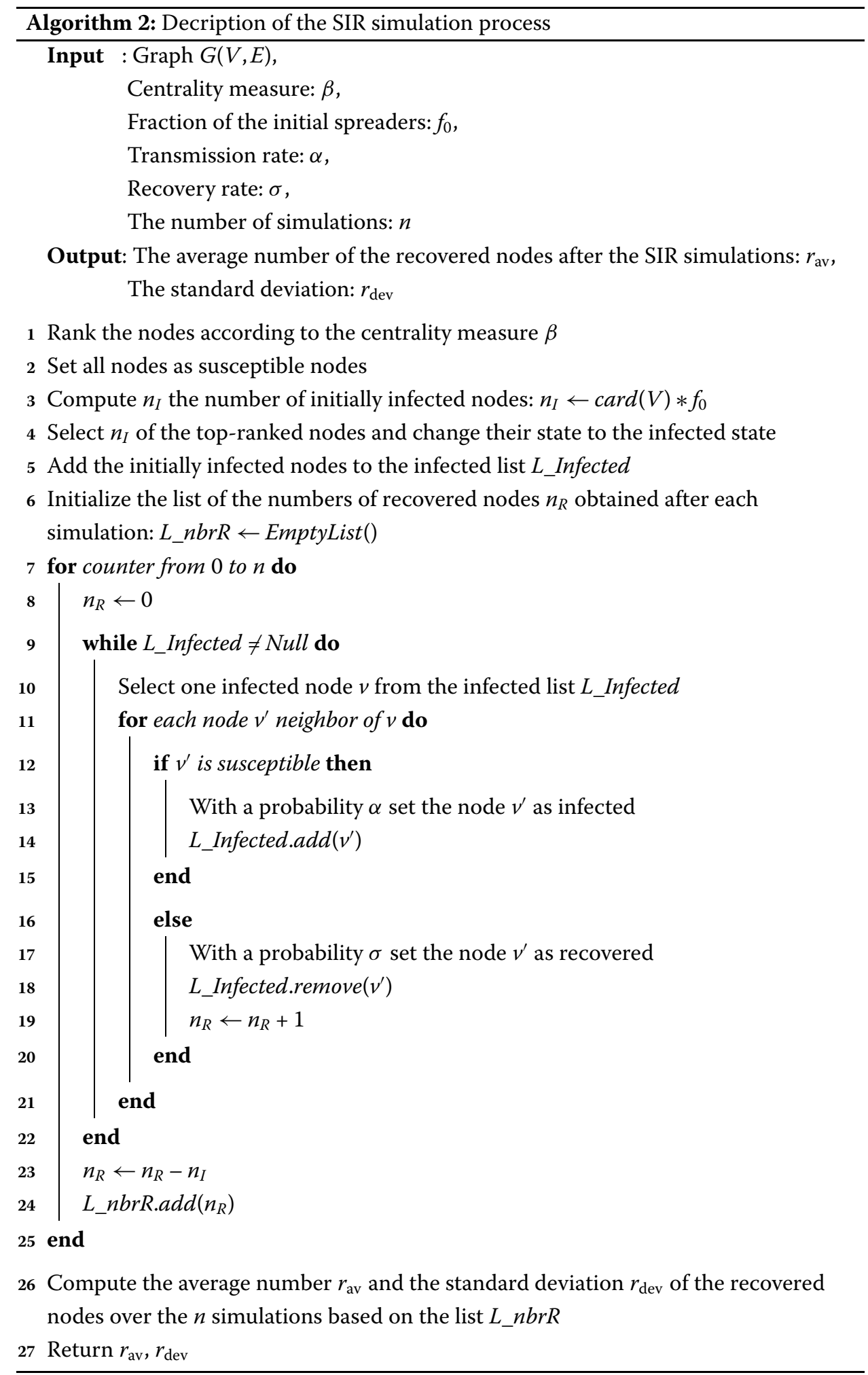

of the SIR simulations are averaged over 200 independent realizations. A more detailed description of the SIR simulation process is given in Algorithm 2. 


\subsection{Evaluation criteria}

In order to compare the ranking efficiency of a centrality measure with the one obtained by a reference centrality measure, we compute the relative difference of the outbreak size. It is given by:

$$
\Delta r=\frac{R_{m}-R_{s}}{R_{s}},
$$

where $R_{m}$ is the final number of recovered nodes of the ranking method under test, and $R_{s}$ is the final number of recovered nodes for the reference method. Thus, a positive value of $\Delta r$ indicates a higher efficiency of the method under test as compared to the reference.

\section{Experimental results}

Extensive experiments have been performed in order to evaluate the effectiveness of the most popular Modular centrality extensions (Degree, Betweenness, Closeness and Eigenvector centrality) as compared to their standard definition. First, the Local and Global component of the various Modular centrality measures is compared to their standard counterpart. Next, the three ranking methods based on the combination of the components of the Modular centrality are also evaluated. These experiments are conducted on both synthetic and real-world networks.

\subsection{Synthetic networks}

Networks with different mixing parameter values have been generated in order to better understand the effect of the community structure strength on the performance of the various centrality measures. Figure 2 represents the relative difference of the outbreak size as a function of the fraction of immunized nodes with the standard measure used as a reference. The mixing parameter values $\mu$ cover all the range of community structure strength.

\subsubsection{Evaluation of the local and the global component of the Modular centrality}

a. Well-defined community structure In networks with well-defined community structure, the Local component of the Modular centrality always outperforms the standard measures for all the centrality measures as it is shown in the left panels of the Fig. 2 (when $\mu=0.1$ ). The gain is around $20 \%$ as compared to the standard measure for Closeness, Degree and Eigenvector centrality. The smallest gain is for Betweenness centrality with an average value of $10 \%$. On the contrary, the Global component of the Modular centrality is always less performing than the standard measures. These results clearly demonstrate that it is more efficient to immunize the influential nodes inside the communities when there are few inter-community links in the networks. Indeed, as there are few inter-community edges, the infection may die out before reaching other communities. So, the local influence of nodes is more important than global influence in networks with strong community structure.

b. Community structure with medium cohesiveness The middle panels of Fig. 2 show the performance of the various ranking methods in networks with community structure of medium strength $(\mu=0.4)$. In this case, both the Global and Local components of the Modular centrality are always more efficient than the standard centrality. The gain in performances of the Global component of the Modular centrality is always greater than for the 


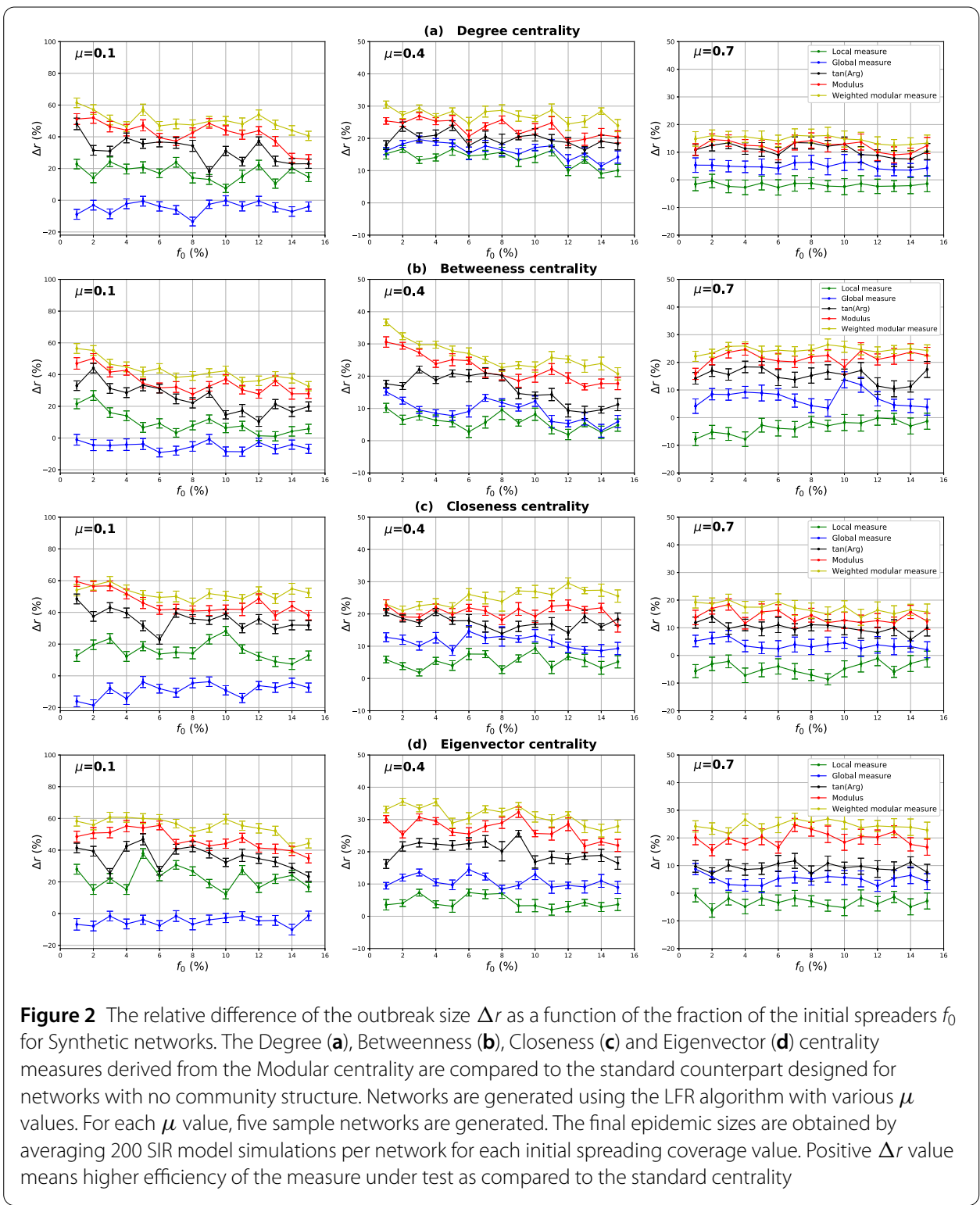

Local component. Indeed, the Global component outperforms the standard measure with a Gain around 12\% for Betweenness, Closeness and Eigenvector centrality. The largest gain is for the Degree centrality with an average value of $17 \%$. The Local component of the Modular centrality performs better than the standard measure with a gain around $5 \%$ for Betweenness, Closeness and Eigenvector centrality and around 12\% for Degree centrality. These results send a clear message: In networks with medium community structure strength, the global influence is more important than the local influence. Indeed, with a greater number of inter-community links, there are more options to spread the epidemics to the other communities of the network.

c. Loose community structure The right panel of Fig. 2 reports the comparison between the Modular centrality and the traditional centrality measures in networks with noncohesive community structure $(\mu=0.7)$. It appears that the relative difference of the out- 
break size between the Global component of the Modular centrality and the standard centrality is always positive while it is always negative for the Local component of the Modular centrality. And this is true for all the centrality measures under test. In fact, there is a gain of around 5\% using the Global component of the Modular centrality, while the Local component performs worse than the traditional measure with an average of $5 \%$ for the Degree, Betweenness, Closeness and Eigenvector centrality measures. Consequently, we can conclude that in networks with a loose community structure the global influence is dominant, even if the difference with the standard measure is not as important than for networks with a medium community structure. Indeed, in this situation $(\mu=0.7)$, the inter-community edges constitute the majority of edges in the network (around 70\% of links lie between the communities). In fact, as the community structure is not well defined, minor differences are observed with a network that has no community structure.

\subsubsection{Evaluation of the ranking methods of the Modular centrality}

Figure 2 reports also the relative difference of the outbreak size $\Delta r$ as a function of the fraction of the initial spreaders $f_{0}$ for the three ranking methods (Modulus and Tangent of the argument of the Modular centrality, Weighted Modular measure) and for the various centrality measure and community structure strength under study. The first observation that can be made from these results is that combining the components of the Modular centrality is always more efficient than using either a single component or the conventional centrality. This remark holds for all the centrality measures studied and whatever the community structure strength. Additionally, the ranking of the three combination methods in terms of efficiency is always the same. The Weighted Modular measure ranks first. It is followed by the Modulus and then the Tangent of the argument of the Modular centrality. We believe that it is due to the fact that the Weighted Modular measure uses more information about the topology of the community structure than its alternatives. Indeed, the weights introduced in this measure allow tuning locally the relative importance of the Local and Global component for each community in the network. Thus, the Weighted Modular measure can adapt to the structure of each community in the network. As a result, it is more efficient than the other proposed ranking methods. One of the main benefits of this result is to highlight the fact that significant gains can result from improving the way the Local and Global component are combined and that there may be still room for improvement in this direction. In other words, even more effective measures can be obtained if relevant additional information about the community structure is used. Furthermore, it is noticed that the ranking strategies show their best performance in networks with a well-defined community structure. For instance, The Modulus of the Modular centrality outperforms the standard measure with a gain, on average, of $40 \%$ in networks with strong community structure, $25 \%$ in networks with community structure of medium strength and $20 \%$ in networks with unclear community structure for all the centrality measures. For the Weighted Modular measure, the gain is around $42 \%$ in networks with strong community structure, $29 \%$ in networks with community structure of medium strength and $25 \%$ in networks with unclear community structure for all the centrality measures. The gain, therefore, decreases as the community structure becomes more and more loose. The reason behind that behavior is that the Local centrality is typical of networks with a community structure while the Global centrality is also a feature of networks with no community structure. As the mixing proportion increases, the differences with networks without community structure 
become less and less important. Indeed, the global network size increases until it tends to represent the major part of the original network. In the limiting case, it is a network with no community structure and the Modular centrality reduces to its Global component which is identical to the classical centrality measures.

\subsection{Real-world networks}

In this section, we report the results of the set of experiments on real-world networks. Experiments performed with synthetic networks have shown that the community structure strength plays a major role in determining the performance of the various centrality measures. Therefore, we adopt the same presentation for real-world networks in order to link the results of this set of experiments with those obtained using synthetic networks. Once the community structure has been uncovered using the Louvain algorithm, the mixing proportion parameter is computed for each network. Estimated values are reported in Table 4. According to these results, we can classify the ego-Facebook network, Power Grid and the ca-GrQc as networks with strong community structure. Princeton, Email-Eu-core and Caltech have a community structure of medium strength while Princeton has a weak community structure.

\subsubsection{Evaluation of the local and global component of the Modular centrality}

a. Well-defined community structure The relative difference of the outbreak size between the community-based measures and the standard measure is reported in Fig. 3. To evaluate their performances in networks with strong community structure, ego-Facebook, power grid and the ca-GrQc networks are used. For these networks, the estimated mixing parameter value ranges from 0.03 to 0.095 . In this figure, we notice that for all the centrality measures under test the standard measure outperforms the Global component of the Modular centrality while it is less performing that its Local component. Let's consider for example the Betweenness centrality. With a fraction of the initial spreaders equal to $8 \%$, the gain in terms of the outbreak size for the Local component of the Modular centrality as compared to the standard Betweenness is $19 \%$ for the ego-Facebook network, $14 \%$ for Power-Grid and 9\% for ca-QrGc. Conversely, in the same situation, the loss associated with the use of the Global component of the Modular centrality instead of the standard Betweenness ranges from $4 \%$ to $11 \%$.

In these networks, communities are densely connected and there are few links lying between the communities. Therefore, in most cases, contagious areas are found in the core of the communities and the spread of the epidemic may stop before even reaching the community perimeter. Thus, there is a low probability that a bridge (inter-community link) propagates the epidemic to the other communities. This is the reason why the Local

Table 4 The estimated mixing parameter $\mu$ and modularity $Q$ of the real-world networks

\begin{tabular}{|c|c|c|c|c|c|c|c|c|}
\hline \multicolumn{2}{|l|}{ Network } & \multicolumn{2}{|c|}{ ego-Facebook } & \multicolumn{2}{|l|}{ Power-grid } & \multicolumn{2}{|l|}{ ca-GrQc } & Princeton \\
\hline$\mu$ & & 0.03 & & 0.034 & & 0.095 & & 0.354 \\
\hline \multirow[t]{4}{*}{ Q } & & 0.834 & & 0.934 & & 0.86 & & 0.753 \\
\hline & Network & & Email-Eu-core & & Caltech & & Georgetown & \\
\hline & $\mu$ & & 0.42 & & 0.448 & & 0.522 & \\
\hline & Q & & 0.569 & & 0.788 & & 0.662 & \\
\hline
\end{tabular}




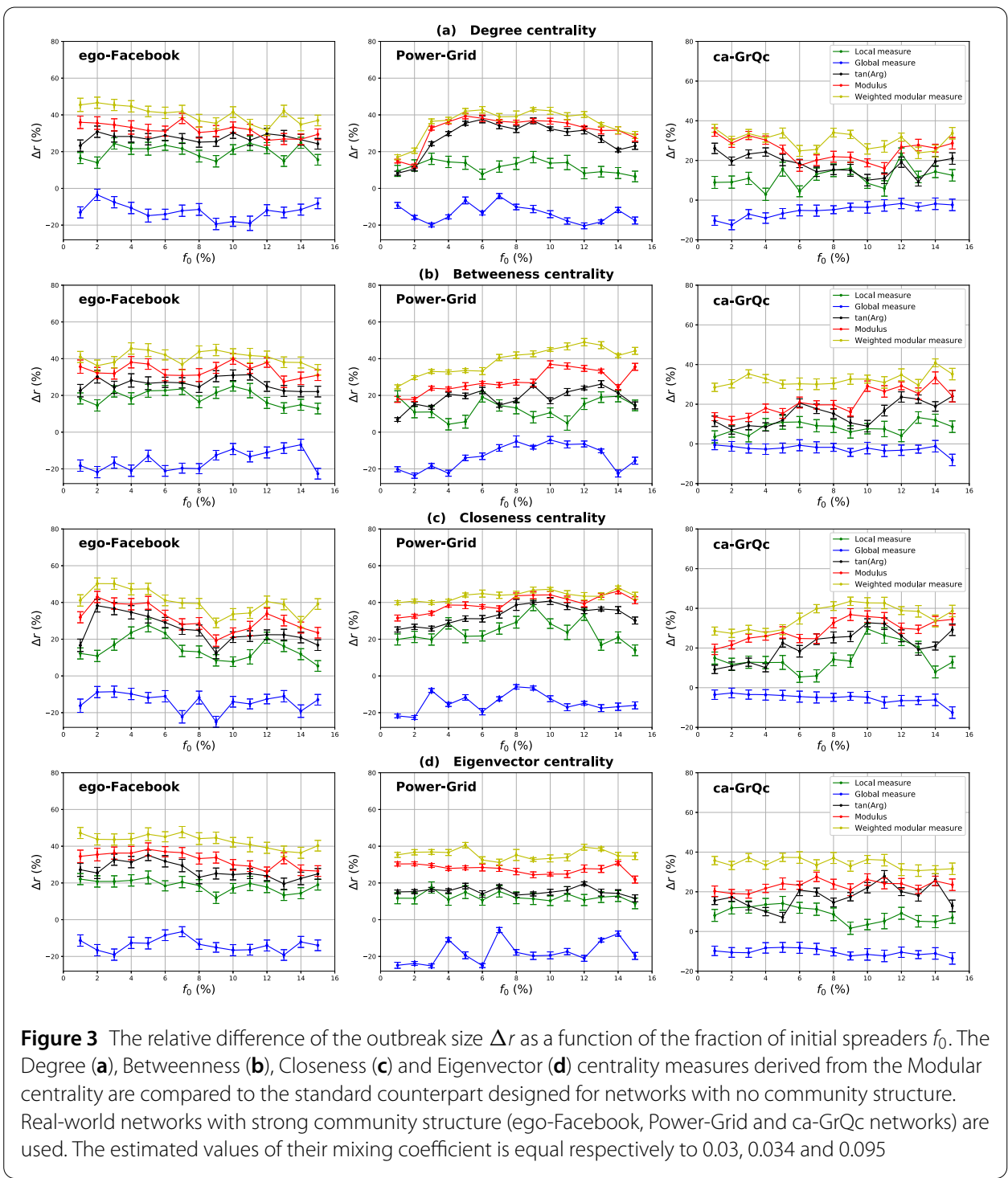

component of the Modular centrality performs always better than the Global component. Furthermore, we can also notice on Fig. 3 that when the mixing parameter value increases (i.e., the community structure gets weaker), the Local component of the Modular centrality gets less efficient while the Global component performs better. This is due to the fact that the Global component increases with the number of inter-community links.

b. Community structure with medium cohesiveness Figure 4 shows the relative difference of the epidemic outbreak size between the community-based measures and the standard centrality measure for real-world networks with medium community structure strength. For that purpose, Princeton, Email-Eu-core and Caltech networks are used since their estimated mixing parameter values range from 0.354 to 0.448 . Results are very clear. In all the situations, both the Local and the Global component of the Modular centrality measures outperform the standard centrality measure. In addition, there is still a slight advantage for the Global component on the Local component. To set these ideas on a simple example, let us consider the Betweenness centrality with an initial fraction of infected nodes 


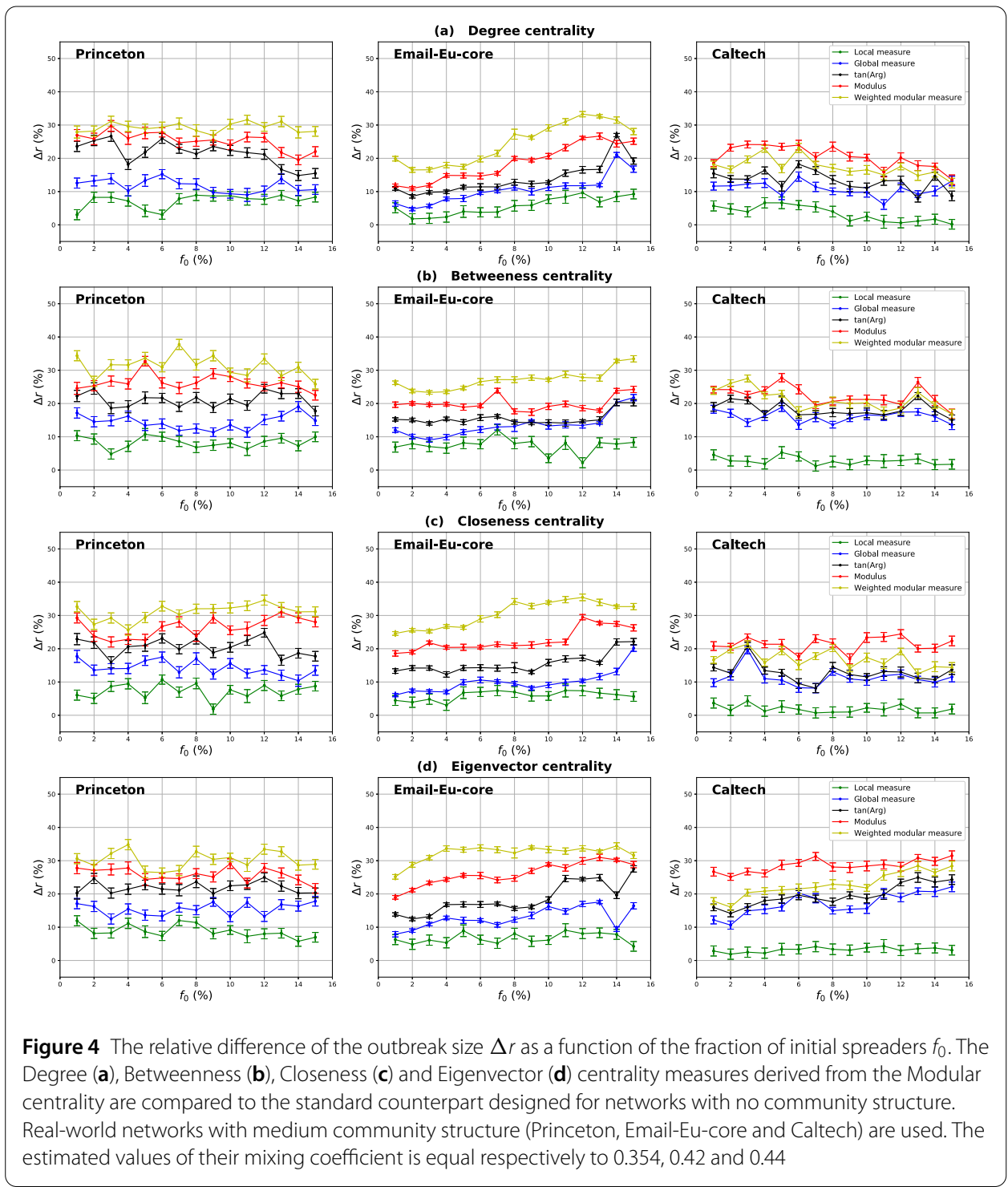

equal to $8 \%$. The Global component of the modular Betweenness measure is more efficient than the traditional Betweenness with a gain of $12 \%$ for the Princeton, $13 \%$ for Email-Eucore and $15 \%$ for the Caltech network. These figures need to be compared to a gain of 8\% for Princeton, 7\% for Email-Eu-core and 3\% for Caltech using the Local component of the Modular Betweenness instead of the classical Betweenness centrality. One can also notice that the gap between their respective performance gets bigger as the value of the mixing parameter increases. Indeed, as the community structure gets weaker, the relative influence of the Global component of the Modular centrality becomes more and more important. In these networks, nodes have approximately as many internal links as there are external links. Therefore, the epidemic can spreads easily to all the communities in the network through the large number of inter-community links. This is the reason why the Global component of the Modular centrality outperforms always the Local component. In addition to that, the community structure of the network is still well preserved, which explains that the Modular centrality is more efficient than the classical centrality. 


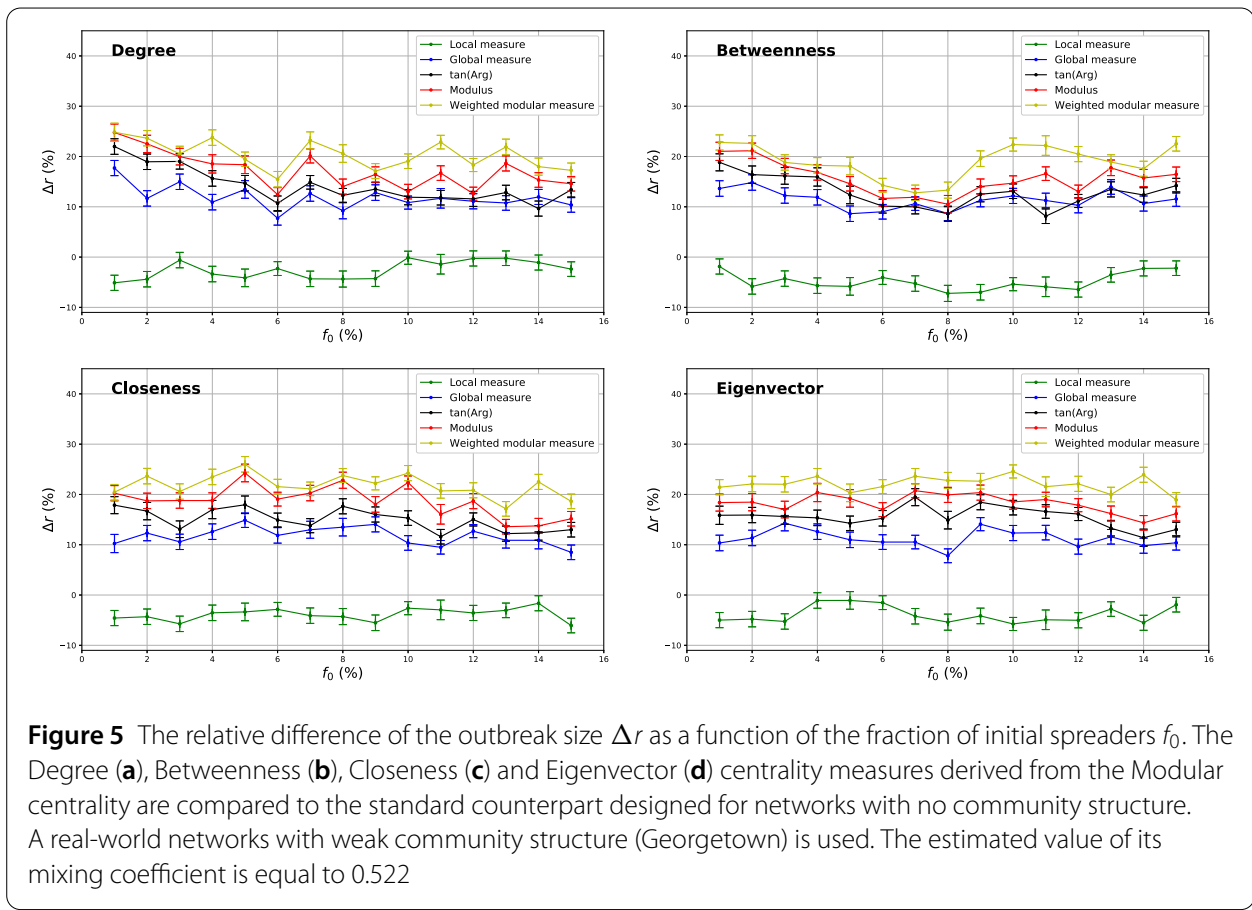

c. Loose community structure Figure 5 shows the relative difference of the epidemic outbreak size between the modular based centrality measures and the standard centrality for the Georgetown network. With a mixing parameter value equal to 0.522 , this network is classified as a network with a weak community structure. In all circumstances, the standard measure performs better than the Local component of the Modular centrality and it performs worse than its Global component. On average, there is a gain of around 10\% for the Global component compared to a loss of $5 \%$ for the Local component of the four centrality measures under test. In this type of networks, the inter-community links predominate, which translates into a greater influence of the Global component of the Modular centrality. Indeed, the epidemic can spread more easily into the various communities of the network through the big amount of external links. Additionally, we notice that the relative difference of the outbreak size between the Global component of the Modular centrality measure and the standard measure decreases as compared to networks with a medium community structure. Indeed, there are less and less topological differences between networks with a weak community structure and networks that have no community structure as the value of the mixing parameter increases.

\subsubsection{Evaluation of the ranking methods of the Modular centrality}

Figures 3 to 5 report also the relative difference of the epidemic outbreak size between the Modular centrality ranking methods and the standard centrality measures. The results are clear evidence of the efficiency of the Modular centrality. Whatever the ranking strategy of the Modular centrality adopted, it outperforms in all the situations the Local and Global component of the Modular centrality and the classical centrality. The improvements in terms of performance compared to classical centrality are quite significant. For instance, with a fraction of initial spreaders equal to $8 \%$, the modulus of the Betweenness Modular centrality allows a gain of $45 \%$ on the ego-Facebook network, $28 \%$ on Princeton and $24 \%$ 
on Georgetown. As the ranking strategies use both the local and the global information of each node, they are more efficient than measures relying on either local or global information taken separately. Furthermore, the Weighted Modular measure is usually the most efficient measure in most cases. It uses the fraction of inter-community links as additional information to target the most influential spreaders in each community. It can give more or less weight to the Local and the Global component according to the individual community structure strength. This explains its superiority over the other ranking measures. To summarize, these experiments reveal that combining the components of the Modular centrality, allows designing efficient ranking methods. In addition, using more relevant information about the community structure at the community level allows designing even more efficient ranking methods. Moreover, the ranking measures exhibit their best results in networks with strong community structure.

\subsubsection{Comparisons with the alternative measures}

Figure 6 shows the average fraction of the epidemic size versus the proportion of the initial spreaders for the Modular centrality components and their standard counterparts. The performance of the alternative Modular centrality measures presented in the related work section is also reported in this figure (i.e., Comm centrality, Number of Neighboring Communities NNC and Community Hub-Bridge centrality CHB). The figures report the results for the Power-grid network in (a) and the Georgetown network in (b). The former has a well-defined community structure while the latter one has a loose community structure. For both networks in Fig. 6(a) and (b) and for all the tested measures, one can see that increasing the proportion of the initial spreaders, the epidemic size increases as well. However, this evolution is slower in Power-grid as compared to Georgetown. Indeed, because of its well-separated modules, the epidemics cannot move easily from one module to another in Power-grid.

Overall, the Betweenness-based centralities (Local, Global, Weighted Modular centrality, Standard Betweenness) outperforms all the other alternatives. Note that, even the standard Betweenness centrality is more efficient than the other alternatives. This result is independent of the community structure strength. In networks with a well-defined community structure (i.e., Power-grid network), one can see on Fig. 6(a) that the Eigenvectorbased centralities are just below, followed by the Degree-based centralities. The lowest performance is obtained by the Closeness-based centrality measures. The Comm and Number of Neighboring Communities exhibit a lower efficiency as compared to the three versions (Standard, Local and Weighted Modular measures) of the four previous centralities. Their performance is, however, as good as the Global measures with slightly higher performance for the NNC measure. These two methods tend to target bridge nodes that have a high global influence in the network. This is the reason why they perform at the same level as global measures. Furthermore, the Community Hub-Bridge measure has globally the same performance as the Weighted Modular Betweenness. As this centrality incorporates both local and global influence of nodes it performs better than most of the other measures. This corroborates the fact that both dimensions must be taken into account in order to design a centrality measure in modular networks.

In networks with a loose community structure (i.e., Georgetown network), one can notice from Fig. 6(b) that the ranks of the centrality measures in terms of efficiency are differ- 


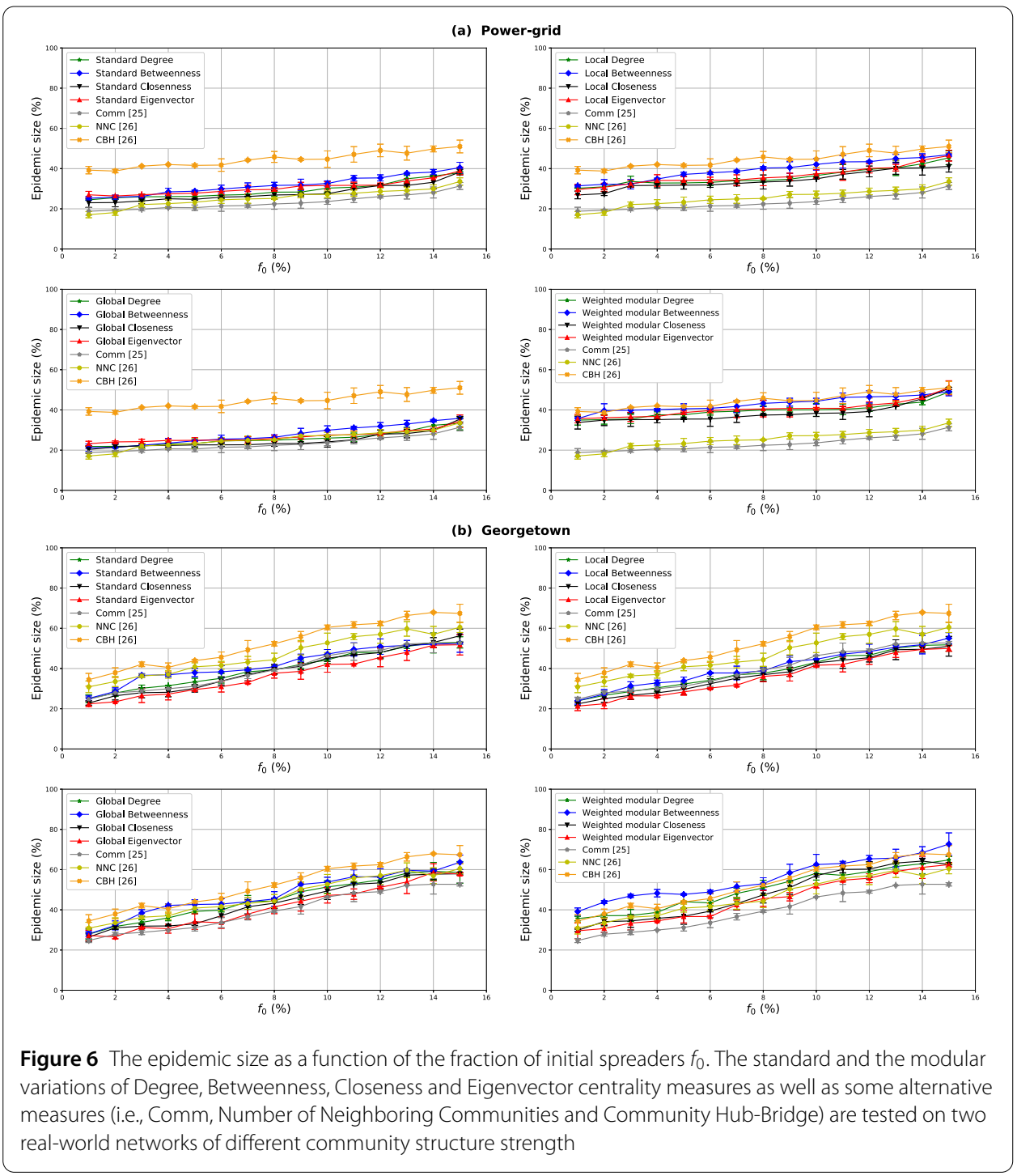

ent. The Betweenness-based measures still rank first, the Degree-based centralities are the second best-performing measures followed by the Closeness and the Eigenvector-based centrality measures. In this type of networks, the Comm and the Number of Neighboring Communities perform better than the Local and the standard centrality measures. Their performance is as good as the Global measures since they highlight also nodes with high global influence. These nodes can play a major role in the spreading process in networks with non-cohesive community structure. This is due to the large amount of inter-community links. Hence the higher performance of these two community-based measures. Additionally, the curves of the Community Hub-Bridge centrality are usually at the top of all the figures. Thus, it is as expected more efficient than the standard and the Modular centrality components of all the tested centralities. It has an overall similar performance that most Weighted Modular measures (Degree, Closeness, Eigenvector), except the Weighted Modular Betweenness which performs better. Both of them targets efficiently nodes with high local and global influence in the network. 
Table 5 The estimated mixing parameter $\mu$ and the modularity $Q$ in Power-grid and Georgetown networks

\begin{tabular}{llll}
\hline Network & Metric & \multicolumn{2}{l}{ Detection algorithm } \\
\cline { 3 - 4 } & & Louvain & Infomap \\
\hline Power-grid & $\mu$ & 0.034 & 0.038 \\
& $\mathrm{Q}$ & 0.92 & 0.93 \\
Georgetown & $\mu$ & 0.522 & 0.491 \\
& $\mathrm{Q}$ & 0.521 & 0.601 \\
\hline
\end{tabular}

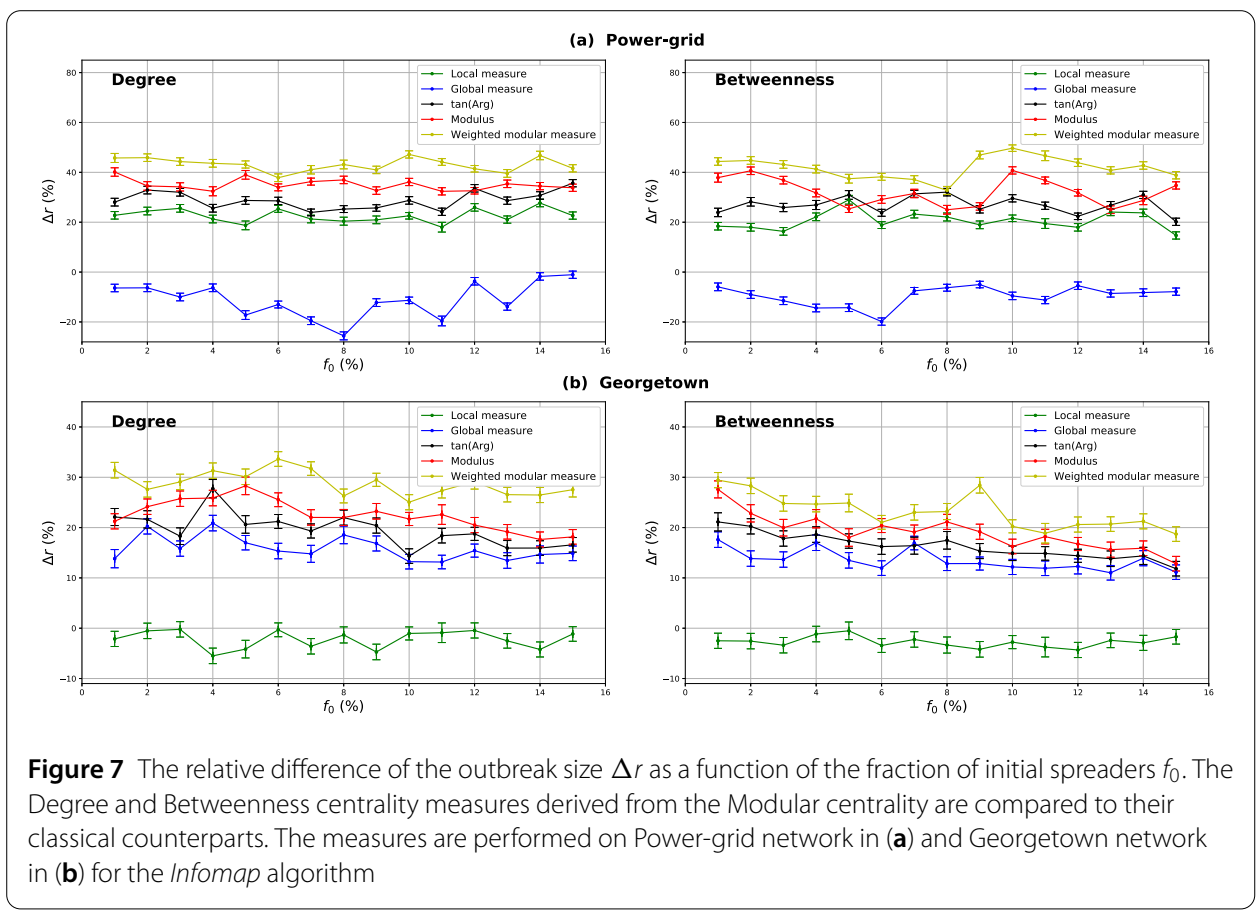

\subsubsection{Influence of the community detection algorithms}

In this section, we report a set of experiments conducted on Power-grid and Georgetown networks using the Infomap algorithm [37] instead of Louvain. These two real-world networks are chosen because of their different community structure strength. The Power-grid network has a strong community structure while Georgetown network has a non-cohesive community structure. The main purpose of these experiments is to get a clear picture of the influence of the community detection algorithm on the performance of the Modular centrality components. The estimated values of the proportion of inter-community links and the modularity for both Infomap and Louvain algorithms are reported in Table 5.

Figure 7 illustrates the relative difference of the epidemic size between the Modular centrality extensions (Degree and Betweenness centrality) with their classical counterparts. It can be inferred from this figure, that the Local, Global and the combination-based methods exhibit overall the same behavior as in the case of the Louvain algorithm. In networks with well-defined community structure (e.g., Power-grid network), the Local measure performs always better than the classical one with an average gain of $23 \%$ and $20 \%$ for the Degree and Betweenness centrality measures respectively. Whereas, the average gain is around $20 \%$ and $19 \%$ in the case of Louvain algorithm for both centrality measures. The standard measure, on the other hand, outperforms the Global measure for all the propor- 
tions of the initial seeds. Additionally, the overall gain of the Weighted Modular measure is around $45 \%$ and $42 \%$ for Degree and Betweenness measures when using Infomap, while it is around $40 \%$ for both centrality measures when Louvain algorithm is employed. Thus, the Modular centrality extensions display a slightly better performance for the Infomap algorithm. Indeed, Infomap and Louvain detection algorithms have nearly the same mixing parameter and modularity measures. Therefore, in networks with a well-defined community structure, both community detection algorithms uncover about the same community structure. Consequently, the performance of the Modular centrality display roughly the same behavior.

In networks with a loose community structure (e.g., Georgetown network), the standard measure is always better performing than the Local measure. The Global measure, however, performs better than the Standard one with an average gain of 17\% and 16\% for the Degree and Betweenness centrality measures respectively. Whereas the average gain is around $11 \%$ in the case of Louvain algorithm for both centrality measures. On the other hand, the overall gain of the Weighted Modular measure is around 29\% and 25\% for Degree and Betweenness measures when using Infomap, while it is around 20\% and $19 \%$ for both centrality measures respectively when Louvain algorithm is employed. In this network, the Infomap algorithm has a relatively smaller mixing parameter and higher modularity. Infomap is then more accurate as compared to Louvain algorithm. That explains why the performance of the Modular centrality components enhances in networks with a non-cohesive community structure when the Infomap detection algorithm is used.

Globally, the results of this set of experiments show that variations of the uncovered community structure impact the performance of the centrality measures. The efficiency of the measures increases with the modularity of the community structure.

\section{Conclusion}

In this paper, we propose a general definition of centrality measures in networks with non-overlapping community structure. It is based on the fact that the intra-community and inter-community links should be considered differently. Indeed, the intra-community edges contribute to the diffusion in localized densely connected areas of the network, while the inter-community links allow the global propagation to the various communities of the network. Therefore, we propose to represent the centrality of modular networks by a two-dimensional vector, where the first component measures the local influence of a node in its community and the second component quantifies its global influence on the other communities. Based on this assumption, centrality measures defined for networks with no-community structure can be easily extended to modular networks. Considering the most influential centrality measures as typical examples, we defined their modular extension. Experiments based on an epidemic spreading scenario using both synthetic and real-world networks have been conducted in order to better understand the influence of the two components of the Modular centrality. First of all, results on synthetic and realworld networks are quite consistent. It appears that the Local component is more effective in networks with a strong community structure while the Global component takes the lead as the community structure gets weaker. Comparison with the classic centrality always turns to the advantage of the Modular centrality. More precisely, in networks with strong community structure, the Local component of the Modular centrality outperforms the Global component and the standard centrality, while in networks with medium or 
weak community structure the Global component performs better than its alternatives. Moreover, it is also observed that combining both components of the Modular centrality in order to rank the nodes according to their influence is always more efficient than to use a single component. Furthermore, a further gain can be obtained if the ranking strategy incorporates more information about community structure strength. We perform also a set of experiments using the Infomap detection algorithm to uncover communities. Results show that the performance of the Modular centrality variants exhibit the same behavior in networks with a well-defined community structure. Their performance, however, is different in networks with a loose community structure. In this case, slightly better results are obtained with the Infomap algorithm.

\section{Acknowledgements \\ Not applicable. \\ Funding \\ Not applicable.}

\section{Abbreviations}

SIR, Susceptible-Infected-Recovered; LFR, Lancichinetti-Fortunato-Radicchi benchmark; GR-QC, General Relativity on Quantum Cosmology.

Availability of data and materials

The datasets used in this article are all publicly available and cited in the references.

\section{Competing interests}

The authors declare that they have no competing interests.

\section{Authors' contributions}

All the authors contributed to designing the proposed method. ZG implemented the model and all the analyses. All authors participated in the formulation and writing of this paper. All authors approved the final manuscript.

\section{Author details}

${ }^{1}$ LRIT URAC No 29, Faculty of Science, Rabat IT Center, Mohammed V University, Rabat, Morocco. ${ }^{2}$ DISP Lab, University of Lyon 2, Lyon, France. ${ }^{3}$ LE2I, UMR6306 CNRS, University of Burgundy, Dijon, France.

\section{Endnotes}

a http://snap.stanford.edu/data

b http://www-personal.umich.edu/ mejn/netdata/

\section{Publisher's Note}

Springer Nature remains neutral with regard to jurisdictional claims in published maps and institutional affiliations.

Received: 13 July 2018 Accepted: 30 April 2019 Published online: 09 May 2019

\section{References}

1. Wang Z, Moreno Y, Boccaletti S, Perc M (2017) Vaccination and epidemics in networked populations - an introduction. Chaos Solitons Fractals 103:177-183

2. Medo M, Zhang Y-C, Zhou T (2009) Adaptive model for recommendation of news. Europhys Lett 88(3):38005

3. Zhang Z-K, Liu C, Zhan X-X, Lu X, Zhang C-X, Zhang Y-C (2016) Dynamics of information diffusion and its applications on complex networks. Phys Rep 651:1-34

4. Coscia M, Giannotti F, Pedreschi D (2011) A classification for community discovery methods in complex networks. Stat Anal Data Min ASA Data Sci J 4(5):512-546

5. Cherifi H (2014) Chapter eleven. Non-overlapping community detection. In: Complex networks and their applications, p 320

6. Fortunato S, Hric D (2016) Community detection in networks: a user guide. Phys Rep 659:1-44

7. Jebabli M, Cherifi H, Cherifi C, Hamouda A (2015) User and group networks on YouTube: a comparative analysis. In: Computer systems and applications (AICCSA), 2015 IEEE/ACS 12th international conference of. IEEE, pp 1-8

8. Jebabli M, Cherifi H, Cherifi C, Hammouda A (2014) Overlapping community structure in co-authorship networks: a case study. In: u- and e-service, science and technology (UNESST), 2014 7th international conference on. IEEE, pp 26-29

9. Palla G, Derényi I, Farkas I, Vicsek T (2005) Uncovering the overlapping community structure of complex networks in nature and society. Nature 435(7043):814

10. Saxena R, Kaur S, Bhatnagar V (2018) Social centrality using network hierarchy and community structure. ArXiv preprint arXiv:1806.08964 
11. Kumar M, Singh A, Cherifi H (2018) An efficient immunization strategy using overlapping nodes and its neighborhoods. In: International World Wide Web Conferences Steering Committee, pp 1269-1275

12. Chakraborty D, Singh A, Cherifi H (2016) Immunization strategies based on the overlapping nodes in networks with community structure. In: International conference on computational social networks. Springer, Berlin, pp 62-73

13. Taghavian F, Salehi M, Teimouri M (2017) A local immunization strategy for networks with overlapping community structure. Phys A, Stat Mech Appl 467:148-156

14. Lü L, Chen D, Ren X-L, Zhang Q-M, Zhang Y-C, Zhou T (2016) Vital nodes identification in complex networks. Phys Rep 650:1-63

15. Ferrara E, De Meo P, Fiumara G, Provetti A (2012) The role of strong and weak ties in Facebook: a community structure perspective. ArXiv preprint arXiv:1203.0535

16. Granovetter MS (1973) The strength of weak ties. Am J Sociol 78:1360-1380

17. Salathé M, Jones JH (2010) Dynamics and control of diseases in networks with community structure. PLoS Comput Biol 6(4):e1000736

18. Gupta N, Singh A, Cherifi H (2015) Community-based immunization strategies for epidemic control. In: Communication systems and networks (COMSNETS), 2015 7th international conference on. IEEE, pp 1-6

19. Gong K, Tang M, Hui PM, Zhang HF, Younghae D, Lai Y-C (2013) An efficient immunization strategy for community networks. PLoS ONE 8(12):e83489

20. Zhao Z, Wang X, Zhang W, Zhu Z (2015) A community-based approach to identifying influential spreaders. Entropy 17(4):2228-2252

21. Nadini M, Sun K, Ubaldi E, Starnini M, Rizzo A, Perra N (2018) Epidemic spreading in modular time-varying networks. Sci Rep 8(1):2352

22. Chan SY, Leung IX, Liò P (2009) Fast centrality approximation in modular networks. In: Proceedings of the 1 st $A C M$ international workshop on complex networks meet information \& knowledge management. ACM, New York, pp 31-38

23. Yoshida T, Yamada Y (2017) A community structure-based approach for network immunization. Comput Intell 33(1):77-98

24. Newman ME (2006) Finding community structure in networks using the eigenvectors of matrices. Phys Rev $E$ 74(3):036104

25. Gupta N, Singh A, Cherifi H (2016) Centrality measures for networks with community structure. Phys A, Stat Mech Appl 452:46-59

26. Ghalmane Z, Hassouni ME, Cherifi H (2018) Immunization of networks with non-overlapping community structure. ArXiv preprint arXiv:1806.05637

27. Luo S-L, Gong K, Kang L (2016) Identifying influential spreaders of epidemics on community networks. ArXiv preprint arXiv:1601.07700

28. Kitromilidis M, Evans TS (2018) Community detection with metadata in a network of biographies of western art painters. ArXiv preprint arXiv:1802.07985

29. Lancichinetti A, Fortunato S, Radicchi F (2008) Benchmark graphs for testing community detection algorithms. Phys Rev E 78(4):046110

30. Orman GK, Labatut V, Cherifi H (2013) Towards realistic artificial benchmark for community detection algorithms evaluation. Int J Web Based Communities 9(3):349-370

31. Orman GK, Labatut V, Cherifi H (2011) On accuracy of community structure discovery algorithms. ArXiv preprint arXiv:1112.4134

32. Orman GK, Labatut V, Cherifi H (2012) Comparative evaluation of community detection algorithms: a topological approach. J Stat Mech Theory Exp 2012(08):P08001

33. Leskovec J, Mcauley JJ (2012) Learning to discover social circles in ego networks. In: Advances in neural information processing systems, pp 539-547

34. Traud AL, Mucha PJ, Porter MA (2012) Social structure of Facebook networks. Phys A, Stat Mech Appl 391(16):4165-4180

35. Moreno Y, Pastor-Satorras R, Vespignani A (2002) Epidemic outbreaks in complex heterogeneous networks. Eur Phys J B, Condens Matter Complex Syst 26(4):521-529

36. Wang W, Liu Q-H, Zhong L-F, Tang M, Gao H, Stanley HE (2016) Predicting the epidemic threshold of the susceptible-infected-recovered model. Sci Rep 6:24676

37. Rosvall M, Bergstrom CT (2008) Maps of random walks on complex networks reveal community structure. Proc Natl Acad Sci 105(4):1118-1123

\section{Submit your manuscript to a SpringerOpen ${ }^{\circ}$ journal and benefit from:}

- Convenient online submission

- Rigorous peer review

- Open access: articles freely available online

- High visibility within the field

- Retaining the copyright to your article

Submit your next manuscript at $>$ springeropen.com 